tissue, while non-invasively tracking stem cells in vivo is a hurdle for its clinical application. Our group has addressed this concern by developing a bioluminescence tomography (BLT) prototype system with micro-CT (MicroCT) registration approach. In this subsequent study, we aimed to assess the mesenchymal stem cells (MSCs) as well as statins by this multimodality imaging platform and other strategies in the model of peripheral arterial disease (PAD).

Methods MSCs were isolated from adipose tissue of the transgenic mice carrying double-fusion reporter genes: firefly luciferase and enhanced green fluorescent protein (Fluc-eGFP). After eGFP flow sorting, $1 \times 10^{7}$ of Fluc-eGFP positive MSCs were injected into the ischaemic hindlimb, created by routine ligation, of the adult nude mice $(n=20)$ with/without rosuvastatin pretreatment. Then we imaged the animals by our 3D BLT and MicroCT modalities as well as a $2 \mathrm{D}$ bioluminescence imaging (BLI). Detailed quantitative reconstruction were performed within the mice by adaptive hp finite element method (hp-FEM). Histological and molecular analysis are used to confirm MSCs' location and angiogenesis.

Results 1 week after engraftment, reconstructed BLT total power in MSCs group was $22.4 \pm 3.1 \mathrm{nW}$, while the $2 \mathrm{D}$ BLI data was $1.7 \times 10^{6} \pm 2.1 \times 10^{5}$ photons $/ \mathrm{s} / \mathrm{cm}^{2} / \mathrm{sr}$. The total power decreased gradually from week 1 to week 6 , from mean $22.4 \mathrm{nW}$ to $1.9 \mathrm{nW}$, demonstrating MSCs' survival and proliferation. The combined treat by MSCs and rosuvastatin exhibited longer signal and higher power of the MSCs $(p<0.01)$, which is also confirmed by 2D BLI with robust correlation $\left(r^{2}=0.93\right)$. Moreover, BLT with MicroCT provided detailed 3D images of MSCs and angiogenesis in the hindlimbs. Immunohistology, RT-PCR and Western Blot showed that MSCs with/without rosuvastatin recovered vessel density in contrast to the control and its signal pathway.

Conclusions Versatile 3D molecular imaging modalities facilitate the super spatiotemporal visualisation and quantification of the MSCs in the ischaemic hindlimb, while MSCs hold beneficial potential for treating $\mathrm{PAD}$ and its survival environment in vivo may be promoted by rosuvastatin.

\section{E0190 STUDY ON THE ROLE OF CD4CD25TREG ON ATHEROSCLEROSIS IN APOE MICE}

doi:10.1136/hrt.2010.208967.190

Li Xuemei, Li Yujie, Zheng Dongdan, Tang Hao, Wen Mingxiang. The First Affiliated Hospital Sun Yatsen University, Guangzhou, China

Objective To evaluate the effectiveness of right ventricular septum (RVS) pacing for the treatment of arrhythmia.

Methods We searched the electronic bibliographic databases, including Cochrane Central Register of Controlled Trials (Issue 1, 2010), PubMed (1994 2010.5). EMBASE (1994 2010.5), CNKI (1994 2010.5), VIP (1994 2010.5), Wanfang database (1994 2010.5) to assemble the randomised controlled trials (RCTs) of RVS Pacing compared with right ventricular apical (RVA) pacing. Two reviewers evaluated the quality of included studies based on the Handbook 5.0.2 and extracted data independently. Meta-analysis was performed by RevMan 5.0 software.

Results 35 RCTs involving 2054 patients were included. The results of meta-analysis showed: compared with the RVA pacing, RVS pacing could significantly reduce the ORS wave duration $(\mathrm{MD}=-0.05,95 \% \mathrm{CI}-0.07$ to -0.02$)$, significantly increase the left ventricular ejection fraction of 3 months and 18 months after operation ( $\mathrm{MD}=7.10,95 \% \mathrm{CI} 3.03$ to 11.17$)$; $(\mathrm{MD}=7.44,95 \% \mathrm{CI}$ 5.46 to 9.42 ). 3 months later, there was no significant difference between the two groups with regard to pacing threshold $(\mathrm{MD}=-13.88$, 95\% CI -29.75 to 2.00$)$, Compared with RVA, RVS was associated with a significant reduction in threshold perception current $(\mathrm{MD}=-0.73,95 \% \mathrm{CI}-29.75$ to -0.12$)$ and impedance $(\mathrm{MD}=-75.12,95 \% \mathrm{CI}-35.53$ to -14.71$)$.
Conclusion RVS pacing can give patients a good physiological state which is more consistent with biventricular electric conduction, and lead to haemodynamic improvement. RVS pacing might be expected to become a preferred site of ventricular pacing.

\section{e0191 FREE FATTY ACIDS INHIBIT THE EXPRESSION OF ANTICOAGULANT THROMBOMODULIN PROTEIN C SYSTEM AN IMPLICATION FOR THE DEVELOPMENT OF THE PROTHROMBOTIC STATE IN METABOLIC SYNDROME}

doi:10.1136/hrt.2010.208967.191

${ }^{1}$ Rong Yuanyuan, ${ }^{1}$ Zhang Mei, ${ }^{2}$ Shen Ying Hu, 'Zhang Yun, ${ }^{2}$ Zhang Lin. ${ }^{1}$ Oilu Hospital Shandong University, Shandong, China; ${ }^{2}$ Baylor College of Medicine, USA

Background Metabolic syndrome displays a significant prothrombotic state which renders patients highly susceptible to myocardial infarction, ischaemic stroke and peripheral vascular diseases. Although many clinical studies showed that the prothrombotic state is a key feature of metabolic syndrome, the pathogenesis and mechanisms involved are not completely understood. Metabolic syndrome is often characterised by obesity and subsequent high circulating concentrations of free fatty acids (FFAs). Thrombomodulin (TM)- protein C system is a key endogenous anticoagulant system. We therefore examined the effects of FFAs on the expression of $\mathrm{TM}$ - protein $\mathrm{C}$ system and the mechanisms involved.

Methods 8 to10 weeks of male wide-type mice were divided into two groups. One group of mice $(n=15)$ were fed a chow diet, while the other group of mice $(n=15)$ were fed a high-fat diet for 20 weeks. Tailbleeding time and the occlusion time induced by $\mathrm{FeCl}_{3}$ were recorded. Circulating free fatty acids were measured. Primary human aortic endothelial cells (HAECs) were cultured and treated with different dose of palmitic acid (PA). TM expression and protein $\mathrm{C}$ activation were measured with western blot and RT-PCR. The effects of JNK, p38 stress pathways and transcriptional factor Foxo1 involved in PA-inhibited TM expression were elucidated with siRNA-induced gene silencing and DNA plasmids-induced gene over-expression.

Results Mice fed a high-fat diet showed notably higher blood level of FFAs $(p<0.05)$, and a shortened tail-bleeding time and a shortened occlusion time induced by $\mathrm{FeCl}_{3}(\mathrm{p}<0.01)$. Furthermore, TM was down-regulated in obese mice $(p<0.001)$. PA significantly decreased the expression of TM and protein $\mathrm{C}$ activation in a dose-dependent fashion $(p<0.01)$. Silencing JNK and p38 with specific siRNAs decreased PA-induced thrombomodulin suppression $(p<0.01)$, while wide type of JNK plasmid can enhance PA's inhibitory effect on TM expression $(p<0.05)$. When endothelial cells were transiently transfected with Foxo1-specific siRNAs, PA-induced inhibition of TM expression was significantly decreased $(p<0.001)$. Furthermore, wide type and constitutively active of Foxo1 plasmids enhanced PA's inhibitory effect of TM expression $(p<0.05)$.

Conclusion In summary, a high-fat diet induced high elevated circulating FFAs level, and the prothrombotic state in mice. PA inhibited the expression of TM and the activation of protein $C$ in endothelial cells. JNK, p38/Foxo1 pathway mediated free fat acids' inhibitory effect of TM expression, which may be a new therapeutic target in treating and reducing cardiovascular and cerebrovascular complications of the metabolic syndrome.

\section{Q0192 A METAANALYSIS OF RIGHT VENTRICULAR SEPTUM PACING}

doi:10.1136/hrt.2010.208967.192

Yulong Zhang, Feng Bai, Xiaowei Zhang, Hui Pan.

Objective To evaluate the effectiveness of right ventricular septum (RVS) pacing for the treatment of arrhythmia. 